\title{
Patrones de innovación en las empresas hosteleras: el caso español
}

\section{Patterns of innovation in hospitality firms: the spanish case}

\author{
M. ${ }^{a}$ Concepción LóPez Fernández ${ }^{1}$ \\ Ana M. ${ }^{a}$ Serrano Bedia ${ }^{1}$ \\ RAQUEL GÓMEZ LÓPEZ ${ }^{1}$ \\ Universidad de Cantabria
}

Recibido el 6 de julio de 2009, aceptado el 22 de marzo de 2010

$\mathrm{N}^{\mathrm{o}}$ de clasificación JEL: M1, L83

DOI: $10.5295 / \mathrm{cdg} .100166 \mathrm{ml}$

Reseña bibliográfica: LÓPEZ, M. C.; SERRANO, A. M. y GÓMEZ, R. (2011): "Patrones de innovación en las empresas hosteleras: el caso español", Cuadernos de Gestión, Vol 11, n 1, pp.59-74, DOI: $10.5295 / \mathrm{cdg} .100166 \mathrm{ml}$

\section{Resumen:}

El objetivo de este trabajo es contribuir a la mejora del conocimiento existente sobre los patrones de innovación en las empresas hosteleras. Para ello, se ha utilizado una muestra representativa de 443 empresas hosteleras españolas pertenecientes al CNAE-55, que forman parte de la Tercera Encuesta de Innovación Tecnológica elaborada por la Comisión Europea (CIS-3) la cual se basa en una versión revisada del Manual de Oslo. Para la consecución del objetivo planteado se ha llevado a cabo, en primer lugar, un análisis descriptivo que muestra evidencia empírica de varios aspectos relacionados con las actividades de innovación de las empresas hosteleras. En segundo lugar, se ha realizado un análisis multivariante, en particular un análisis factorial y un análisis cluster, que nos ha permitido identificar 5 patrones de innovación existentes entre las empresas hosteleras españolas: "empresas altamente innovadoras en proceso", "empresas altamente innovadoras en producto", "empresas tradicionales orientadas al proceso", "empresas innovadoras con escasa cooperación" y "empresas innovadoras con una fuerte cooperación".

Palabras clave:

empresas hosteleras, patrones de innovación, innovación tecnológica, CIS-3.

\begin{abstract}
:
The aim of this paper is to contribute to the understanding of innovation patterns in hospitality firms. To this end, we have use a representative sample of 443 Spanish hospitality firms (NACE-55) taken from the Third Community Innovation Survey (CIS-3), which is based on the revised version of the Oslo Manual. For the attainment of the objective of the paper we carry out, on the first place, a descriptive analysis to provide empirical
\end{abstract}

\footnotetext{
${ }^{1}$ Departamento de Administración de Empresas, Universidad de Cantabria, Los Castros s/n, 39005 Santander, España, lopezm@unican.es; serranoa@unican.es; gomezlr@unican.es
} 
evidence on a variety of aspects of innovation activities in hospitality firms. On the second place, we carry out a multivariate analysis and, in particular factor and cluster analysis to identify innovation patterns. The results confirm the existence of 5 clusters or patterns of innovation among hospitality firms that we have called "high process-innovating firms", "high product-innovating firms", "traditional process-oriented firms", "innovative low cooperated firms", and "innovative strongly cooperated firms".

\section{Key Words:}

hospitality firms, innovation patterns, technological innovation, CIS-3. 


\section{INTRODUCCIÓN}

Los servicios han sido considerados tradicionalmente en la literatura como un sector poco innovador dominado por la tecnología de sus proveedores (Pavitt, 1984). Sin embargo, existen varios trabajos empíricos que han tratado de demostrar que el sector servicios es mucho más activo en cuanto a innovación de lo que popularmente se piensa (Hipp et al., 2000; Coombs y Miles, 2000). En esta línea, a medida que aumenta la disponibilidad de datos sobre innovación en el sector servicios han ido surgiendo en la literatura más intentos de clasificación del mismo de acuerdo con sus patrones de innovación. Así, Miozzo y Soete $(2001)^{2}$ desarrollan una taxonomía para las empresas de servicios distinguiendo tres grupos: 1) "dominado por los proveedores" que incluye los servicios públicos y sociales como la educación y los servicios personales como la hostelería; 2) "intensivos en producción, intensivos en escala y servicios de redes" y 3) "proveedores especializados en tecnología y empresas de base científica".

Esta taxonomía ha sido contrastada empíricamente en varios trabajos (Evangelista, 2000³; Hollestein, 2003; Camacho y Rodríguez, 2008). El estudio empírico sobre patrones de innovación llevado a cabo por Evangelista (2000) para las empresas de servicios italianas muestra la existencia de cuatro tipos de empresas a las que denomina "usuarios de tecnologías", "de base científica y tecnológica", "interactivos y basados en tecnologías de la información" y "consultoría técnica". Las empresas hosteleras forman parte del grupo "interactivos y basados en tecnologías de la información” cuyo patrón innovador característico refleja tanto el papel central de la adaptación al consumidor o customization, como la importancia de aprender haciendo o learning by doing. Dentro de este grupo, bancos, seguros y hostelería presentan una escasa intensidad innovadora y conceden una gran importancia a la adquisición de maquinaria y a la interacción con los clientes.

Un estudio similar fue desarrollado por Hollestein (2003) para el sector suizo de los servicios. El autor identificó 5 clusters: "empresas de base científica y altamente tecnológicas totalmente integradas", "empresas desarrolladoras de redes integradas orientadas a las TI (Tecnologías de la Información)", "empresas con innovaciones incrementales orientadas al mercado y con débiles enlaces externos", "innovadoras en proceso orientadas al coste y con fuertes enlaces externos a lo largo de la cadena de valor" y "empresas de escaso perfil innovador sin apenas enlaces externos". Este último cluster engloba a los subsectores menos innovadores como los servicios personales, la hostelería, la venta al por menor y el transporte.

Más recientemente, siguiendo esta línea de investigación, Camacho y Rodríguez (2008) analizan el sector servicios español e identifican 5 clusters: "empresas innovadoras con estrategias relacionadas con la eficiencia productiva", "empresas innovadoras en producto", "empresas escasamente innovadoras y dominadas por los proveedores", "empresas altamente innovadoras y escasamente integradas" y "empresas altamente innovadoras y

\footnotetext{
${ }^{2}$ Una versión preliminar de este artículo es el working paper 89-31 del Maastricht Economic Research Institute on Innovation and Technology-MERIT (Soete y Miozzo, 1989).

${ }^{3}$ Una versión preliminar de este artículo se presentó en la VIII Annual RESER Conference (Evangelista y Savona, 1998).
} 
fuertemente integradas". Los autores clasifican a las empresas hosteleras como empresas escasamente innovadoras y dominadas por los proveedores.

El presente trabajo está estrechamente relacionado con los estudios mencionados anteriormente pero difiere de ellos en un aspecto principal, ya que está centrado en el estudio de un subsector particular del sector servicios como es la hostelería. La elección del subsector hostelero como objeto de estudio se debe principalmente a la importancia del mismo dentro del sector turístico, así como a la disponibilidad de microdatos para esta actividad en particular.

Teniendo en cuenta estas consideraciones, el objetivo de este trabajo es contribuir a mejorar el conocimiento existente sobre los patrones de innovación en las empresas hosteleras. Para la consecución de dicho objetivo se ha llevado a cabo, en primer lugar, un análisis descriptivo que muestra evidencia empírica de varios aspectos relacionados con las actividades de innovación de las empresas hosteleras. En segundo lugar, se ha realizado un análisis multivariante, en particular un análisis factorial y un análisis cluster que permiten, por un lado, reducir un amplio conjunto de indicadores de innovación en factores y, por otro, realizar grupos homogéneos de empresas en función de dichos factores. Para ello, se ha utilizado una muestra representativa de 443 empresas hosteleras españolas pertenecientes al CNAE-55, que forman parte de la Tercera Encuesta de Innovación Tecnológica elaborada por la Comisión Europea (CIS-3) la cual se basa en una versión resisada del Manual de Oslo (OECD, 1997).

Para alcanzar el objetivo propuesto, el resto del trabajo se organiza como sigue. La sección segunda proporciona un análisis descriptivo de la actividad innovadora de las empresas hosteleras. En la tercera sección se presentan los resultados del análisis factorial y el cluster. La última sección recoge los principales resultados y conclusiones de nuestro estudio.

\section{ANÁLISIS DESCRIPTIVO}

\subsection{Tipos de innovación}

A la hora de analizar la innovación en el sector servicios es importante distinguir entre innovación tecnológica e innovación no tecnológica, ya que este último tipo de innovación es el más común en las empresas de servicios según la literatura (Gallouj y Weinstein, 1997; Tether y Storey, 1998; Gallouj, 2002). El CIS-3 define la innovación tecnológica como un producto nuevo o sensiblemente mejorado, un bien o servicio introducido en el mercado o la introducción en la empresa de un proceso nuevo o sensiblemente mejorado. A su vez, la innovación no tecnológica comprende varios cambios estratégicos y organizativos como la puesta en práctica de estrategias corporativas nuevas o sensiblemente cambiadas, de técnicas de gestión avanzadas, de estructuras organizativas nuevas o sensiblemente mejoradas, cambios importantes en la estrategia de marketing y cambios significativos en la apariencia estética o en el diseño, u otros cambios subjetivos en algún producto. La Tabla 1 muestra el porcentaje de empresas hosteleras, empresas pertenecientes al resto del sector servicios y empresas industriales que realizan innovación tecnológica y no tecnológica. 
Tabla 1

Tipos de innovación (porcentaje)

\begin{tabular}{lccc}
\hline \multicolumn{1}{c}{ Sectores } & Total empresas & Innovación tecnológica & Innovación no tecnológica \\
\hline Hostelería & 443 & 12,4 & 30,3 \\
Sector servicios & 4.335 & 26,3 & 39,0 \\
Industria & 6.399 & 41,8 & 36,8 \\
\hline
\end{tabular}

Fuente: CIS-3

Los resultados nos permiten confirmar que las empresas hosteleras realizan innovación tecnológica en menor medida que el sector servicios y la industria. Como era de esperar, el sector servicios es el que lleva a cabo una mayor innovación no tecnológica, lo cual está en línea con los resultados obtenidos por varios trabajos (Miles, 2005) y puede deberse a las peculiaridades de dicho sector (Sirilli y Evangelista, 1998): a) la estrecha interacción entre producción y consumo (co-terminalidad) que dificulta la diferenciación tradicional entre innovación en producto y en proceso; b) el contenido intensivo en información de las actividades de servicios y de producción; c) el papel fundamental de los recursos humanos como factor básico de competitividad; d) la importancia de los cambios organizativos en la introducción de nuevas tecnologías, así como un medio para producir y ofrecer nuevos servicios.

\subsection{Fuentes de innovación}

Respecto a las fuentes de innovación es necesario especificar si el esfuerzo innovador de las empresas se centra en el desarrollo de la I+D interna o si, por el contrario, se basa principalmente en la adquisición de maquinaria y equipos.

Tabla 2

\section{Gastos en innovación}

\begin{tabular}{lcccc}
\hline \multirow{2}{*}{ Sectores } & \multicolumn{4}{c}{ Gasto en innovación dirigido a (\%) } \\
\cline { 2 - 5 } & $\begin{array}{c}\text { I+D } \\
\text { Interna }\end{array}$ & $\begin{array}{c}\text { I+D } \\
\text { Externa }\end{array}$ & Adquisición de maquinaria & $\begin{array}{c}\text { Otros } \\
\text { gastos }\end{array}$ \\
\hline Hostelería & 2,8 & 5,9 & 39,6 & 51,7 \\
Sector servicios & 25,5 & 6,1 & 32,8 & 35,6 \\
Industria & 33,6 & 6,3 & 39,2 & 20,9 \\
\hline
\end{tabular}

Fuente: CIS-3 
De acuerdo con la Tabla 2, la característica más importante de las empresas hosteleras es el escaso gasto en I+D interna como fuente de innovación. Con respecto al sector servicios y a la industria, los resultados ponen de manifiesto que aunque el sector servicios realiza I+D interna en menor grado que la industria, esta diferencia es únicamente del $8 \%$, mientras que la diferencia entre las empresas hosteleras y el sector servicios es aproximadamente del 23\%. Respecto al resto de fuentes de innovación las diferencias son mínimas y podemos afirmar que las empresas hosteleras centran su esfuerzo innovador en la adquisición de maquinaria.

\subsection{Objetivos de innovación y Fuentes de información para la innovación}

Las Tablas 3 y 4 muestran la importancia que dan las empresas a los objetivos relacionados con la introducción de innovaciones y a las fuentes de información para la innovación, respectivamente.

Tabla 3

Objetivos de innovación.

Porcentaje de empresas para las cuales el objetivo es muy importante

\begin{tabular}{lccc}
\hline \multicolumn{1}{c}{ Objetivos } & Hostelería & Sector servicios & Industria \\
\hline Ampliar la gama de servicios & 12,7 & 30,1 & 32,7 \\
Incrementar la cuota de mercado & 7,3 & 19,9 & 22,5 \\
Mejorar la calidad & 47,3 & 46,2 & 43,8 \\
Mejorar la flexibilidad de producción & 14,5 & 21,5 & 25,8 \\
Mejorar la capacidad de producción & 16,4 & 24,5 & 34,3 \\
Reducir costes laborales & 9,1 & 13,4 & 16,1 \\
Reducir costes materiales & 3,6 & 6,6 & 10,8 \\
Desarrollar productos compatibles con el medioambiente & 16,4 & 12,9 & 23,2 \\
Cumplir reglamentos y normas & 29,1 & 21,3 & 29,5 \\
\hline
\end{tabular}

Fuente: CIS-3

La Tabla 3 muestra que la mejora de la calidad es un objetivo muy importante para un amplio porcentaje de empresas hosteleras (47,3\%), el sector servicios $(46,2 \%)$ y la industria $(43,8 \%)$. Estos resultados están en línea con los obtenidos por Sirilli y Evangelista (1998). Otros objetivos relevantes para las empresas hosteleras son el cumplimiento de reglamentos y normas, la mejora de la capacidad de producción y el desarrollo de productos compatibles con el medioambiente. 
Tabla 4

Fuentes de información para la innovación.

Porcentaje de empresas para las cuales la fuente es muy importante

\begin{tabular}{lccc}
\hline \multicolumn{1}{c}{ Fuentes } & Hostelería & Sector servicios & Industria \\
\hline Fuentes internas & 36,4 & 48,3 & 42,1 \\
Otras empresas del grupo & 21,8 & 21,0 & 14,9 \\
Proveedores & 25,5 & 28,3 & 24,9 \\
Clientes & 14,5 & 22,1 & 22,7 \\
Competidores & 9,1 & 14,1 & 11,1 \\
Universidades e institutos de educación superior & 9,1 & 5,7 & 4,7 \\
Centros tecnológicos públicos & 3,6 & 7,2 & 6,4 \\
Conferencias, seminarios, revistas especializadas, etc & 14,5 & 12,6 & 9,8 \\
Ferias y exhibiciones & 18,2 & 10,0 & 17,5 \\
\hline
\end{tabular}

Nota: El CIS-3 para España no hace distinción entre las distintas "fuentes internas".

Fuente: CIS-3

Los resultados que se muestran en la Tabla 4 nos permiten concluir que las fuentes internas son las fuentes de información para la innovación más importantes para los tres grupos de la muestra. Entre las fuentes externas, destacan los proveedores ya que son muy importantes para un $25-28 \%$ de las empresas. Por lo que respecta a las universidades, los institutos de educación superior y los centros tecnológicos públicos podemos decir que son percibidos como fuentes de información relevantes para una minoría de las empresas de los tres grupos analizados.

\section{ANÁLISIS MULTIVARIANTE}

Para llevar a cabo el análisis multivariante se ha constituido una muestra con 55 empresas hosteleras que han respondido a las preguntas del CIS-3 relacionadas con la innovación. Dicho análisis se ha ejecutado en dos pasos. El primer paso consiste en la realización de un análisis factorial exploratorio con el objeto de reducir el número de indicadores de innovación utilizados en el estudio. En el segundo paso utilizamos los factores extraídos anteriormente para identificar grupos de empresas de acuerdo a su distancia y proximidad en relación a esos factores. Existen varios estudios que aplican un enfoque similar tanto para servicios como para industria (Pomares, 1998; Sellenthin y Hommen, 2002; Hollestein 2003; Camacho y Rodríguez, 2008)

\subsection{Análisis factorial}

Siguiendo el trabajo reciente de Camacho y Rodríguez (2008), se ha llevado a cabo un análisis factorial con 24 indicadores de innovación que se recogen en la Tabla 5. Todas las varia- 
bles son cualitativas de tipo binario $(1=$ si y $0=$ no $) \mathrm{u}$ ordinal con cuatro niveles de respuesta ( 3 = importancia alta, $2=$ importancia media, $1=$ importancia baja y $0=$ nada importante).

TABLA 5

Indicadores de innovación empleados en el análisis factorial de las empresas hosteleras

\begin{tabular}{|c|c|c|c|}
\hline Indicadores & Descripción & Escala & Valor \\
\hline \multicolumn{4}{|c|}{ Tipos de innovación } \\
\hline Inprod & Innovación en producto & Nominal (si/no) & 1,0 \\
\hline Inproc & Innovación en proceso & Nominal (si/no) & 1,0 \\
\hline \multicolumn{4}{|l|}{ Fuentes de innovación } \\
\hline $\mathrm{I}+\mathrm{D}$ interna & I+D interna & Nominal (si/no) & 1,0 \\
\hline I+D externa & I+D externa & Nominal (si/no) & 1,0 \\
\hline Maquinaria & Maquinaria y equipos & Nominal (si/no) & 1,0 \\
\hline Tecnología & Tecnología inmaterial & Nominal (si/no) & 1,0 \\
\hline \multicolumn{4}{|c|}{ Objetivos de la innovación } \\
\hline Gama & Ampliar la gama de servicios & Ordinal & $3-0$ \\
\hline Cuotamcdo & Incrementar la cuota de mercado & Ordinal & $3-0$ \\
\hline Calidad & Mejorar la calidad & Ordinal & $3-0$ \\
\hline Flexprod & Mejorar la flexibilidad de producción & Ordinal & $3-0$ \\
\hline Capprod & Mejorar la capacidad de producción & Ordinal & $3-0$ \\
\hline Costlab & Reducir los costes laborales & Ordinal & $3-0$ \\
\hline Costmat & Reducir los costes materiales & Ordinal & $3-0$ \\
\hline Prodamb & $\begin{array}{l}\text { Desarrollar productos compatibles } \\
\text { con el medioambiente }\end{array}$ & Ordinal & $3-0$ \\
\hline Regulación & Cumplir reglamentos y normas & Ordinal & $3-0$ \\
\hline \multicolumn{4}{|c|}{ Fuentes de información para la innovación } \\
\hline Intfuentes & Fuentes internas & Ordinal & $3-0$ \\
\hline Empgrupo & Otras empresas del grupo & Ordinal & $3-0$ \\
\hline Proveedores & Proveedores & Ordinal & $3-0$ \\
\hline Clientes & Clientes & Ordinal & $3-0$ \\
\hline Competidores & Competidores & Ordinal & $3-0$ \\
\hline Universidades & Universidades & Ordinal & $3-0$ \\
\hline Centrostecno & Centros tecnológicos públicos & Ordinal & $3-0$ \\
\hline Conferencias & Conferencias & Ordinal & $3-0$ \\
\hline Ferias & Ferias y exhibiciones & Ordinal & $3-0$ \\
\hline
\end{tabular}

Fuente: Elaboración propia. 
Con el objeto de sintetizar la información que se muestra en la Tabla 5 se ha realizado un análisis factorial exploratorio cuyo test de esfericidad de Barlett (276 nivel de significatividad 0,000$)$ e índice de Kayser-Meyer-Olkin $(0,631)$ nos permite confirmar la validez de los datos. Mediante el análisis de componentes principales se han obtenido 8 factores que explican un total del 75,2\% de la varianza. Una vez que los factores han sido rotados utilizando el método varimax, las dimensiones identificadas se recogen en la Tabla 6.

Tabla 6

\section{Factores extraídos utilizando el método varimax}

\begin{tabular}{|c|c|c|c|c|c|c|c|c|}
\hline & Factor 1 & Factor 2 & Factor 3 & Factor 4 & Factor 5 & Factor 6 & Factor 7 & Factor 8 \\
\hline Ferias & 0,821 & 0,221 & 0,041 & 0,014 & 0,148 & 0,201 & 0,077 & 0,124 \\
\hline Cuotamcdo & 0,816 & 0,06 & 0,146 & $-0,211$ & 0,068 & $-0,042$ & $-0,049$ & $-0,193$ \\
\hline Conferencias & 0,749 & 0,128 & 0,101 & 0,084 & 0,239 & 0,275 & 0,024 & 0,084 \\
\hline Gama & 0,671 & 0,185 & 0,148 & $-0,462$ & 0,194 & $-0,158$ & $-0,014$ & $-0,058$ \\
\hline Calidad & 0,459 & 0,369 & 0,396 & $-0,153$ & 0,182 & $-0,249$ & 0,164 & $-0,179$ \\
\hline Capprod & 0,155 & 0,852 & 0,031 & 0,011 & 0,121 & 0,06 & 0,129 & $-0,094$ \\
\hline Costlab & 0,101 & 0,791 & 0,08 & $-0,031$ & $-0,007$ & 0,044 & 0,087 & 0,109 \\
\hline Flexprod & 0,09 & 0,736 & 0,412 & 0,103 & $-0,006$ & $-0,064$ & $-0,015$ & 0,002 \\
\hline Costmat & 0,093 & 0,735 & 0,013 & 0,171 & 0,091 & 0,281 & $-0,007$ & 0,033 \\
\hline Clientes & 0,169 & 0,024 & 0,729 & $-0,246$ & 0,306 & 0,155 & $-0,043$ & $-0,126$ \\
\hline Empgrupo & $-0,128$ & 0,169 & 0,691 & $-0,172$ & 0,186 & 0,113 & 0,282 & 0,17 \\
\hline Competidores & 0,3 & 0,185 & 0,688 & $-0,058$ & 0,094 & 0,204 & $-0,205$ & $-0,21$ \\
\hline Inproc & $-0,073$ & 0,167 & $-0,178$ & 0,778 & 0,097 & $-0,117$ & $-0,099$ & 0,174 \\
\hline Inprod & 0,422 & 0,054 & 0,184 & $-0,773$ & $-0,017$ & $-0,037$ & $-0,088$ & 0,087 \\
\hline Proveedores & 0,286 & 0,195 & 0,474 & 0,504 & $-0,151$ & 0,026 & 0,048 & 0,252 \\
\hline Regulación & 0,17 & 0,145 & 0,171 & 0,02 & 0,886 & 0,065 & 0,078 & $-0,097$ \\
\hline Prodamb & 0,237 & 0,011 & 0,173 & 0,014 & 0,882 & 0,118 & $-0,051$ & 0,098 \\
\hline Universidades & 0,15 & 0,121 & 0,368 & $-0,067$ & 0,073 & 0,744 & $-0,127$ & $-0,072$ \\
\hline Centrostecno & 0,128 & 0,355 & 0,113 & 0,023 & 0,282 & 0,732 & $-0,197$ & 0,006 \\
\hline Intfuentes & 0,29 & 0,225 & 0,349 & 0,077 & 0,202 & $-0,453$ & $-0,081$ & 0,354 \\
\hline $\mathrm{I}+\mathrm{D}$ interna & $-0,13$ & 0,123 & 0,069 & $-0,16$ & 0,052 & $-0,213$ & 0,737 & 0,155 \\
\hline Maquinaria & 0,191 & 0,248 & $-0,014$ & 0,502 & $-0,044$ & 0,061 & 0,664 & $-0,129$ \\
\hline Tecnología & 0,471 & $-0,093$ & $-0,08$ & 0,064 & $-0,027$ & $-0,103$ & 0,55 & 0,303 \\
\hline I+D externa & $-0,027$ & 0,017 & $-0,067$ & 0,091 & $-0,013$ & $-0,057$ & 0,163 & 0,881 \\
\hline$\%$ Varianza & 26,12 & 12,23 & 9,77 & 7,09 & 6,49 & 4,58 & 4,42 & 4,21 \\
\hline
\end{tabular}

Fuente: Elaboración propia. 
El primer factor, "orientación al mercado" está formado por los resultados indirectos de la innovación en producto: ampliación de la gama de productos, mejorar la calidad e incrementar la cuota de mercado, así como la interacción indirecta de las empresas por medio de ferias y conferencias. El segundo factor, "eficiencia productiva", recoge todos los objetivos relacionados con la eficiencia del proceso productivo: reducción de costes laborales y materiales y la mejora de la flexibilidad y capacidad productiva. El tercer factor llamado "fuentes de información de mercado" se refiere a la información ofrecida por otras empresas del grupo así como por competidores y clientes. El cuarto factor, "tipos de innovación y proveedores" agrupa tanto la innovación en producto y en proceso como la información suministrada por los proveedores. El quinto factor, "innovación responsable" muestra los objetivos de la actividad innovadora relativos al desarrollo de productos compatibles con el medioambiente y el cumplimiento de reglamentos y normas. El sexto factor, "cooperación y fuentes internas de información" hace referencia a la cooperación de la empresa con universidades y centros públicos de investigación y la importancia que las empresas dan a las fuentes internas de información como fuentes de innovación. El séptimo factor, "fuentes de innovación" refleja la participación de la empresa en los diferentes tipos de actividades innovadoras excluyendo la I+D externa, la cual se incluye en el octavo y último factor.

\subsection{Análisis cluster}

El análisis factorial nos ha ofrecido un número reducido de factores no relacionados entre sí que nos permite realizar el análisis cluster evitando errores. En este caso y utilizando los 8 factores identificados en el paso anterior, hemos optado por un análisis cluster jerárquico (con el método de Ward y la distancia euclídea al cuadrado) debido al limitado número de empresas que forman la muestra. El resultado de dicho análisis son 5 grupos. Para facilitar su interpretación, en la Tabla 7 se detalla la relación entre las empresas que forman cada cluster y los indicadores que forman cada factor, así como la media del porcentaje de la cifra de negocios debida a la innovación que presenta cada cluster.

Tabla 7

Clusters de las empresas hosteleras e indicadores de innovación ordenados por factores (porcentajes)

\begin{tabular}{clccccc}
\hline Factores & Indicadores de innovación & Cluster 1 & Cluster 2 & Cluster 3 & Cluster 4 & Cluster 5 \\
\hline Factor 1 & Orientación al mercado & & & & & \\
& $\begin{array}{l}\text { Ampliar gama de servicios } \\
\text { Incrementar cuota de }\end{array}$ & 0 & 10 & 11,1 & 0 & 12,5 \\
& mercado & 16,7 & 10 & 5,6 & 0 & 12,5 \\
& Mejorar la calidad & 33,3 & 70 & 38,9 & 38,5 & 62,5 \\
& Conferencias y revistas & 0 & 10 & 22,2 & 0 & 37,5 \\
& Ferias y exhibiciones & 16,7 & 20 & 22,2 & 0 & 37,5
\end{tabular}




\begin{tabular}{|c|c|c|c|c|c|c|}
\hline Factores & Indicadores de innovación & Cluster 1 & Cluster 2 & Cluster 3 & Cluster 4 & Cluster 5 \\
\hline \multirow[t]{5}{*}{ Factor 2} & Eficiencia productiva & & & & & \\
\hline & $\begin{array}{l}\text { Mejorar la flexibilidad de } \\
\text { producción }\end{array}$ & 33,3 & 60 & 16,7 & 7,7 & 50 \\
\hline & $\begin{array}{l}\text { Mejorar la capacidad de } \\
\text { producción }\end{array}$ & 0 & 10 & 27,8 & 0 & 37,5 \\
\hline & Reducir costes laborales & 16,7 & 10 & 11,1 & 7,7 & 0 \\
\hline & Reducir costes materiales & 0 & 0 & 11,1 & 0 & 0 \\
\hline \multirow[t]{4}{*}{ Factor 3} & $\begin{array}{l}\text { Fuentes de información } \\
\text { de mercado }\end{array}$ & & & & & \\
\hline & Otras empresas del grupo & 16,7 & 50 & 11,1 & 23,1 & 12,5 \\
\hline & Clientes & 0 & 20 & 5,6 & 15,4 & 37,5 \\
\hline & Competidores & 0 & 20 & 0 & 0 & 37,5 \\
\hline \multirow[t]{4}{*}{ Factor 4} & $\begin{array}{l}\text { Tipos de innovación y } \\
\text { proveedores }\end{array}$ & & & & & \\
\hline & Innovación en producto & 33,3 & 100 & 27,8 & 61,5 & 62,5 \\
\hline & Innovación en proceso & 100 & 60 & 88,9 & 38,5 & 62,5 \\
\hline & Proveedores & 66,7 & 30 & 22,2 & 0 & 37,5 \\
\hline \multirow[t]{3}{*}{ Factor 5} & Innovación responsable & & & & & \\
\hline & $\begin{array}{l}\text { Desarrollar productos } \\
\text { compatibles con el } \\
\text { medioambiente }\end{array}$ & 33,3 & 20,0 & 16,7 & 0 & 25 \\
\hline & $\begin{array}{l}\text { Cumplimiento de } \\
\text { reglamentos y normas }\end{array}$ & 33,3 & 40 & 38,9 & 7,7 & 25 \\
\hline \multirow[t]{4}{*}{ Factor 6} & $\begin{array}{l}\text { Cooperación y fuentes } \\
\text { internas de información }\end{array}$ & & & & & \\
\hline & Universidades & 0 & 0 & 0 & 0 & 0 \\
\hline & $\begin{array}{l}\text { Centros públicos de } \\
\text { investigación }\end{array}$ & 0 & 0 & 0 & 0 & 25 \\
\hline & Fuentes internas & 66,7 & 70 & 44,4 & 7,7 & 0 \\
\hline \multirow[t]{4}{*}{ Factor 7} & Fuentes de innovación & & & & & \\
\hline & I+D interna & 50 & 0 & 5,6 & 38,5 & 0 \\
\hline & Maquinaria y equipos & 83,3 & 20 & 83,3 & 38,5 & 75,0 \\
\hline & Tecnología inmaterial & 66,7 & 10 & 38,9 & 7,7 & 12,5 \\
\hline \multirow[t]{4}{*}{ Factor 8} & $I+D$ externa & & & & & \\
\hline & I+D externa & 100 & 10 & 0 & 0 & 0 \\
\hline & Resultados** & 0 & 21,3 & 4 & 0,2 & 4,4 \\
\hline & $\mathrm{N}^{\circ}$ de Empresas & 6 & 10 & 18 & 13 & 8 \\
\hline
\end{tabular}

**La variable resultados es la media del porcentaje de la cifra de negocios debida a la innovación en cada empresa.

Fuente: Elaboración propia. 
Podemos definir el Cluster 1 como "empresas hosteleras altamente innovadoras en proceso", es decir, empresas que principalmente desarrollan innovaciones en proceso, ya que únicamente el 33\% de las empresas hosteleras de este cluster realizan innovaciones en producto, mientras que el $100 \%$ de las mismas desarrollan innovaciones en proceso. Para estas empresas, todas las fuentes de innovación son inputs esenciales para la innovación. Entre las diferentes fuentes de información, excepto las fuentes internas, la única fuente que juega un papel principal son los proveedores. Por tanto, podemos afirmar que este cluster es similar al denominado por Pavitt (1984) como "dominado por los proveedores".

El Cluster 2 incluye a las "empresas hosteleras altamente innovadoras en producto". Estas empresas presentan altos niveles de innovación en producto $\mathrm{y}$, al igual que ocurre en el Cluster 5, muestran los niveles más bajos de I+D interna, siendo escasamente utilizadas el resto de fuentes de innovación. Este cluster sitúa como principales objetivos de la innovación la mejora de la calidad y de la flexibilidad de producción y el cumplimiento de reglamentos y normas por encima del resto de los clusters. Finalmente, respecto a las fuentes de información para la innovación cabe destacar el importante papel tanto de las fuentes internas como de las empresas del mismo grupo.

El Cluster 3 está formado por "empresas hosteleras tradicionales orientadas al proceso". En este grupo, el porcentaje de empresas que desarrollan innovación en proceso es de un $88,9 \%$. El esfuerzo innovador se concentra en la adquisición de maquinaria y de tecnología inmaterial. El desarrollo de I+D interna no es muy común. Este cluster asigna una alta importancia a la mejora de la calidad y de la capacidad de producción. Estas empresas se centran tanto en la reducción de costes laborales y materiales, como en el cumplimiento de reglamentos y normas. Con respecto a las fuentes de información, las fuentes internas seguidas por los proveedores y las conferencias juegan un papel destacado.

En el Cluster 4 se agrupan las "empresas hosteleras innovadoras con escasa cooperación”. Dentro de este grupo, el porcentaje de empresas hosteleras que desarrollan innovación en producto es de un $65,1 \%$, mientras que el 38,5\% de las empresas desarrollan innovaciones en proceso. Para estas empresas, la I+D interna y la adquisición de maquinaria y equipos son inputs esenciales para la innovación. Entre los diferentes objetivos de innovación, estas empresas se centran en la mejora de la calidad del producto. Como fuentes de información más relevantes se encuentran otras empresas del mismo grupo seguidas por las fuentes internas, aunque cabe destacar que las empresas hosteleras de este cluster no establecen ningún tipo de cooperación con universidades, centros de investigación públicos, proveedores y competidores, y tampoco acuden a conferencias y ferias.

El Cluster 5 abarca a las "empresas hosteleras innovadoras con una fuerte cooperación". Son empresas que llevan a cabo tanto innovación en producto como en proceso basándose principalmente en la adquisición de maquinaria y equipos. Los objetivos de innovación más importantes son la mejora de la calidad y de la flexibilidad y capacidad de producción y, en menor medida, el desarrollo de productos compatibles con el medioambiente. Las fuentes de información para la innovación como proveedores, clientes, competidores, conferencias y ferias presentan el mismo nivel de importancia para las empresas hosteleras de este cluster. Es importante mencionar que las empresas que forman este cluster son las únicas que cooperan con centros públicos de investigación.

Si se analiza el porcentaje de la cifra de negocios debida a la innovación que presenta cada cluster (variable Resultados de la Tabla 7) se pueden destacar varias cuestiones. La 
primera cuestión relevante es que la decisión de innovar, fundamentalmente en producto/ servicio, unida a la elección de actividades exclusivamente externas con el objeto de perseguir la mejora de calidad y la flexibilidad del servicio es la que permite obtener un mayor rendimiento, medido como porcentaje de la cifra de negocios debida a la innovación, a gran distancia del resto de los clusters analizados. Por el contrario, las empresas fuertemente orientadas a la innovación en proceso y con alta actividad de I+D interna son las que muestran los peores resultados. Por último, la opción de combinar esfuerzos innovadores tanto en producto como en proceso recurriendo a la cooperación con otras entidades es una estrategia que se ha mostrado asimismo efectiva entre las empresas analizadas.

\section{CONCLUSIONES}

Este trabajo ha investigado los patrones de innovación de las empresas hosteleras españolas. Para la consecución de este objetivo se ha llevado a cabo, en primer lugar, un análisis descriptivo cuyos principales resultados se pueden resumir como sigue: 1) la innovación no tecnológica es el tipo de innovación más común en las empresas hosteleras; 2) la compra de maquinaria y equipos es la fuente de innovación más importante para las empresas de la muestra; 3) de los objetivos de innovación, la mejora de la calidad destaca como el más relevante y 4) la información para innovar es obtenida principalmente de departamentos internos de producción/distribución, proveedores, otras empresas del grupo, ferias y exhibiciones.

En segundo lugar, se ha realizado un análisis factorial y un análisis cluster jerárquico que nos ha permitido demostrar la existencia de 5 grupos que confirman la presencia de patrones de innovación muy diferentes entre las empresas hosteleras. Esta circunstancia refuerza el interés de realizar un trabajo centrado en un único subsector, la hostelería en este caso, por cuanto se confirma que el mismo no se comporta de forma homogénea, sino que pueden identificarse comportamientos empresariales claramente diferenciados dentro del mismo.

Como aspectos a destacar del análisis cluster realizado, aproximadamente la mitad de las empresas hosteleras españolas analizadas opta bien por la innovación en producto $(42 \%)$ o bien por la innovación en proceso (44\%), siendo las empresas hosteleras que realizan ambos tipos conjuntamente una minoría (15\%). Además, se confirma que la realización de actividades de I+D como vía para el desarrollo de innovaciones es relativamente escasa, dado que sólo un 29,1\% de las empresas hosteleras de la muestra optan por la realización de actividades de I+D externas para el desarrollo de sus actividades de innovación, porcentaje que se sitúa en el 16,36\% en el caso de la I+D interna, y únicamente el 5,5\% de las empresas hosteleras combinan las dos vías para la realización de actividades de I+D.

En cuanto a la efectividad de las estrategias innovadoras desarrolladas por las empresas del sector, los resultados obtenidos indican que las empresas hosteleras con mejores resultados son las que orientan su actividad innovadora al logro del objetivo de mejora de la calidad y de flexibilidad en la producción/prestación del servicio, antes que a la reducción de costes, especialmente de personal. Este resultado parece lógico en un contexto como el español, en el que resulta difícil competir con los destinos turísticos emergentes únicamente en clave de costes, siendo necesario buscar una vía de diferenciación como puede ser la 
centrada en la calidad y el servicio, tal y como ha venido intentando desarrollar el sector en los últimos años. Por otro lado, señalar asimismo que la clave del éxito innovador en las empresas hosteleras españolas no parece estar tanto en la realización de actividades de I+D por la vía interna, sino en combinar el acceso a la innovación tecnológica por la vía externa, o incluso la cooperación, con actividades de innovación no tecnológica -organizativa, o comercial- a partir del aprovechamiento de todas las fuentes de información al alcance de la empresa. En esta línea, la posibilidad de acceder al conocimiento de las empresas que pertenecen a un mismo grupo empresarial -cadena, $u$ otra fórmula de alianza/cooperación- a través, por ejemplo, de informes, documentos de trabajo, bases de datos de clientes parece ser, en el caso del sector hostelero español, un factor asimismo relevante de cara al éxito de su actividad innovadora.

Los resultados anteriores pueden resultar de interés para directivos y responsables del sector, por cuanto podrían ayudar a orientar mejor la elección de la estrategia innovadora por parte de las empresas hosteleras españolas. No obstante, cabe señalar que se trata de un primer estudio de naturaleza exploratoria, que deberá ser ampliado y confirmado en futuras investigaciones.

\section{REFERENCIAS BIBLIOGRÁFICAS}

CAMACHO, J.A. Y RODRIGUEZ, M. (2008): «Patterns of innovation in the service sector: some insights from the Spanish Innovation Survey?». Economics of Innovation and New Technology. 17(5), pp. 459-471.

COOMBS, R. Y MILES, I. (2000): «Innovation, measurement and services: the new problematic». En Metcalfe, S.J. y Miles, I. (Ed), Innovation Systems in the Service Sectors. Measurement and Case Study Analysis. Kluwer Academic Publishers, Boston. 85-104.

EVANGELISTA, R. Y SAVONA, M. (1998): «Patterns of Innovation in Services: The Results of the Italian Innovation Survey». Ponencia presentada al 7th Annual RESER Conference. Berlin.

EVANGELISTA, R. (2000): «Sectoral patterns of technological change in services». Economics of Innovation and New Technologies, 9, pp. 183-221.

GALLOUJ, F. Y WEINSTEIN, O. (1997): «Innovation in services». Research Policy, 26(4-5), pp. 537-556.

GALLOUJ, F. (2002): «Innovation in the Service Economy. The new wealth of nations». Edward Elgar. Cheltenham. UK. Pp. xv y ss.

HIPP, C., TETHER, B. S. Y MILES, I. (2000): «The Incidence and Effects of Innovation in Services; Evidence from Germany». International Journal of Innovation Management, 4(4), pp. 417-454.

HOLLENSTEIN, H. (2003): «Innovation modes in the Swiss service sector: a cluster analysis based on firm-level data». Research Policy, 32(5), pp. 845-863.

MILES, I. (2005): «Innovation in Services». En Fagerberg, J.; Mowery, D.C. y Nelson, R.R. The Oxford Handbook of Innovation. Oxford University Press. Oxford. UK.

MIOZZO, M. Y SOETE, L. (2001): «Internationalization of services: a technological perspective». Technological Forecasting and Social Change, 67(2/3), pp.159-185.

OECD (1997): «The Measurement of Scientific and Technological Activities». Proposed guidelines for collecting and interpreting technological innovation data. Oslo Manual. OECD. Paris.

PAVITT, K. (1984): «Sectoral patterns of technical change: towards a taxonomy and a theory». Research Policy, 13(6), pp. 343-373. 
POMARES, I. (1998): «El comportamiento de las empresas innovadoras en Andalucía: Aplicación de técnicas de análisis multivariante». Economía industrial, 319, pp. 141-150.

SELLENTHIN, M.O. Y HOMMEN, L. (2002): «How innovative is Swedish industry? A factor and cluster analysis». International review of Applied Economics, 16, pp. 319-331.

SIRILLI, G. Y EVANGELISTA, R. (1998): «Technological innovation in services and manufacturing: results from Italian surveys». Research Policy, 27, pp. 881-899.

SOETE, L. Y MIOZZO, M. (1989): «Trade and Development in Services: A Technological Perspective». Working Paper $n^{\circ}$ 89-031, MERIT. Maastricht.

TETHER, B.S. Y STOREY, D.J. (1998): «Smaller Firms and Europe's High Technology Sectors: A Framework for Analysis and Some Statistical Evidence». Research Policy, 26(9), pp. 947-971. 
